\title{
Nucleon form factors in dispersion theory
}

\author{
H.-W. Hammer ${ }^{\mathrm{a}}$ \\ Helmholtz-Institut für Strahlen- und Kernhysik (Theorie), Universität Bonn, Nussallee 14-16, D-53115 Bonn, Germany \\ / \\ Published online: 11 May 2006 - C Società Italiana di Fisica / Springer-Verlag 2006
}

\begin{abstract}
Dispersion relations provide a powerful tool to analyse the electromagnetic form factors of the nucleon in both the space-like and the time-like regions with constraints from other experiments, unitarity, and perturbative QCD. We give a brief introduction into dispersion theory for nucleon form factors and present first results from our ongoing form factor analysis. We also calculate the two-pion continuum contribution to the isovector spectral functions drawing upon the new high statistics measurements of the pion form factor by the CMD-2, KLOE, and SND collaborations.
\end{abstract}

PACS. 11.55.Fv Dispersion relations - 13.40.Gp Electromagnetic form factors - 14.20.Dh Protons and neutrons

\section{Introduction}

The electromagnetic form factors of the nucleon offer a unique window on strong interaction dynamics over a wide range of momentum transfers $[1,2]$. At small momentum transfers, they are sensitive to the gross properties of the nucleon like the charge and magnetic moment, while at high momentum transfers they encode information on the quark substructure of the nucleon as described by QCD.

Their detailed understanding is important for unraveling aspects of perturbative and nonperturbative nucleon structure. The form factors also contain important information on nucleon radii and vector meson coupling constants. Moreover, they are an important ingredient in a wide range of experiments from Lamb shift measurements [3] to measurements of the strangeness content of the nucleon [4].

With the advent of the new continuous beam electron accelerators such as CEBAF (Jefferson Lab.), ELSA (Bonn), and MAMI (Mainz), a wealth of precise data for space-like momentum transfers has become available [5]. Due to the difficulty of the experiments, the time-like form factors are less well known. While there is a fair amount of information on the proton time-like form factors $[6,7$, $8,9,10]$, only one measurement of the neutron form factor from the pioneering FENICE experiment [11] exists.

It has been known for a long time that the pion plays an important role in the long-range structure of the nucleon [12]. This connection was made more precise using dispersion theory in the 1950's [13,14]. Subsequently, Frazer and Fulco have written down partial-wave dispersion relations that relate the nucleon electromagnetic structure to pion-nucleon $(\pi N)$ scattering and predicted

\footnotetext{
a e-mail: hammer@phys.washington.edu
}

the existence of the $\rho$-resonance $[15,16]$. Despite this success, the central role of the $2 \pi$ continuum in the isovector spectral function has often been ignored. Höhler and Pietarinen pointed out that this omission leads to a gross underestimate of the isovector radii of the nucleon [17]. They first performed a consistent dispersion analysis of the electromagnetic form factors of the nucleon [18] including the $2 \pi$ continuum derived from the pion form factor and $\pi N$-scattering data [19]. In the mid-nineties, this analysis has been updated by Mergell, Meißner, and Drechsel [20] and was later extended to include data in the time-like region $[21,22]$. Recently, the new precise data for the neutron electric form factor have been included as well [23].

Using chiral perturbation theory (ChPT), the longrange pionic structure of the nucleon can be connected to the Goldstone boson dynamics of QCD [24]. The nonresonant part of the $2 \pi$ continuum is in excellent agreement with the phenomenological analysis [25] and the $\rho$ meson contribution can be included as well [26,27,28]. It is well known that vector mesons play an important role in the electromagnetic structure of the nucleon, see, e.g., refs. $[15,29,30,31,32,33]$, and the remaining contributions to the spectral function have usually been approximated by vector meson resonances.

A new twist to this picture was recently given by Friedrich and Walcher [34]. They interpreted the form factor data based on a phenomenological fit with an ansatz for the pion cloud using the idea that the proton can be thought of as virtual neutron-positively charged pion pair. A very long-range contribution to the charge distribution in the Breit frame extending out to about $2 \mathrm{fm}$ was found and attributed to the pion cloud. This was shown to be in conflict with the phenomenologically known $2 \pi$ continuum and ChPT by Hammer, Drechsel, and Meißner [35]. We will address this conundrum in more detail in sect. 9 . 


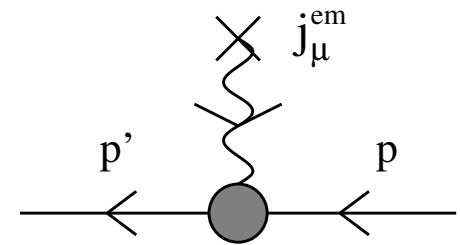

Fig. 1. The nucleon matrix element of the electromagnetic current $j_{\mu}^{\mathrm{em}}$.

In this paper we give a brief introduction into dispersion theory for nucleon form factors and present preliminary results from our ongoing form factor analysis. We also calculate the two-pion continuum contribution to the isovector spectral functions drawing upon the new high statistics measurements of the pion form factor by the CMD-2, KLOE, and SND collaborations. Finally we address the question of the range of the pion cloud and give an outlook on future work.

\section{Definitions}

The electromagnetic (em) structure of the nucleon is determined by the matrix element of the current operator $j_{\mu}^{\mathrm{em}}$ between nucleon states as illustrated in fig. 1 .

Using Lorentz and gauge invariance, this matrix element can be expressed in terms of two form factors,

$$
\left\langle p^{\prime}\left|j_{\mu}^{\mathrm{em}}\right| p\right\rangle=\bar{u}\left(p^{\prime}\right)\left[F_{1}(t) \gamma_{\mu}+i \frac{F_{2}(t)}{2 M} \sigma_{\mu \nu} q^{\nu}\right] u(p),
$$

where $M$ is the nucleon mass and $t=\left(p^{\prime}-p\right)^{2}$ the fourmomentum transfer. For data in the space-like region, it is often convenient to use the variable $Q^{2}=-t>0$. The functions $F_{1}(t)$ and $F_{2}(t)$ are the Dirac and Pauli form factors, respectively. They are normalized at $t=0$ as

$$
F_{1}^{p}(0)=1, F_{1}^{n}(0)=0, F_{2}^{p}(0)=\kappa_{p}, F_{2}^{n}(0)=\kappa_{n},
$$

with $\kappa_{p}=1.79$ and $\kappa_{n}=-1.91$ the anomalous magnetic moments of protons and neutrons in nuclear magnetons, respectively.

It is convenient to work in the isospin basis and to decompose the form factors into isoscalar and isovector parts,

$$
F_{i}^{s}=\frac{1}{2}\left(F_{i}^{p}+F_{i}^{n}\right), \quad F_{i}^{v}=\frac{1}{2}\left(F_{i}^{p}-F_{i}^{n}\right),
$$

where $i=1,2$.

The experimental data are usually given for the Sachs form factors

$$
\begin{aligned}
G_{E}(t) & =F_{1}(t)-\tau F_{2}(t), \\
G_{M}(t) & =F_{1}(t)+F_{2}(t),
\end{aligned}
$$

where $\tau=-t /\left(4 M^{2}\right)$. In the Breit frame, $G_{E}$ and $G_{M}$ may be interpreted as the Fourier transforms of the charge and magnetization distributions, respectively.

The nucleon radii can be defined from the low- $t$ expansion of the form factors,

$$
F(t)=F(0)\left[1+t\left\langle r^{2}\right\rangle / 6+\ldots\right],
$$

where $F(t)$ is a generic form factor. In the case of the electric and Dirac form factors of the neutron, $G_{E}^{n}$ and $F_{1}^{n}$, the expansion starts with the term linear in $t$ and the normalization factor $F(0)$ is dropped.

\section{Dispersion relations and spectral decomposition}

Based on unitarity and analyticity, dispersion relations relate the real and imaginary parts of the electromagnetic (em) nucleon form factors. Let $F(t)$ be a generic symbol for any one of the four independent nucleon form factors. We write down an unsubtracted dispersion relation of the form

$$
F(t)=\frac{1}{\pi} \int_{t_{0}}^{\infty} \frac{\operatorname{Im} F\left(t^{\prime}\right)}{t^{\prime}-t-i \epsilon} \mathrm{d} t^{\prime}
$$

where $t_{0}$ is the threshold of the lowest cut of $F(t)$ (see below) and the $i \epsilon$ defines the integral for values of $t$ on the cut. ${ }^{1}$ Equation (6) relates the em structure of the nucleon to its absorptive behavior.

The imaginary part $\operatorname{Im} F$ entering eq. (6) can be obtained from a spectral decomposition $[13,14]$. For this purpose it is most convenient to consider the em current matrix element in the time-like region $(t>0)$, which is related to the space-like region $(t<0)$ via crossing symmetry. The matrix element can be expressed as

$$
\begin{aligned}
J_{\mu} & =\left\langle N(p) \bar{N}(\bar{p})\left|j_{\mu}^{\mathrm{em}}(0)\right| 0\right\rangle \\
& =\bar{u}(p)\left[F_{1}(t) \gamma_{\mu}+i \frac{F_{2}(t)}{2 M} \sigma_{\mu \nu}(p+\bar{p})^{\nu}\right] v(\bar{p}),
\end{aligned}
$$

where $p$ and $\bar{p}$ are the momenta of the nucleon and antinucleon created by the current $j_{\mu}^{\mathrm{em}}$, respectively. The fourmomentum transfer in the time-like region is $t=(p+\bar{p})^{2}$.

Using the LSZ reduction formalism, the imaginary part of the form factors is obtained by inserting a complete set of intermediate states as $[13,14]$

$$
\begin{aligned}
\operatorname{Im} J_{\mu}= & \frac{\pi}{Z}(2 \pi)^{3 / 2} \mathcal{N} \sum_{\lambda}\left\langle p\left|\bar{J}_{N}(0)\right| \lambda\right\rangle \\
& \times\left\langle\lambda\left|j_{\mu}^{\mathrm{em}}(0)\right| 0\right\rangle v(\bar{p}) \delta^{4}\left(p+\bar{p}-p_{\lambda}\right)
\end{aligned}
$$

where $\mathcal{N}$ is a nucleon spinor normalization factor, $Z$ is the nucleon wave function renormalization, and $\bar{J}_{N}(x)=$ $J^{\dagger}(x) \gamma_{0}$ with $J_{N}(x)$ a nucleon source. This decomposition is illustrated in fig. 2. It relates the spectral function to on-shell matrix elements of other processes.

The states $|\lambda\rangle$ are asymptotic states of momentum $p_{\lambda}$ which are stable with respect to the strong interaction. They must carry the same quantum numbers as the current $j_{\mu}^{\mathrm{em}}: I^{G}\left(J^{P C}\right)=0^{-}\left(1^{--}\right)$for the isoscalar current and $I^{G}\left(J^{P C}\right)=1^{+}\left(1^{--}\right)$for the isovector component of

\footnotetext{
1 The convergence of an unsubtracted dispersion relation for the form factors has been assumed. We could have used a once subtracted dispersion relation as well since the normalization of the form factors is known.
} 


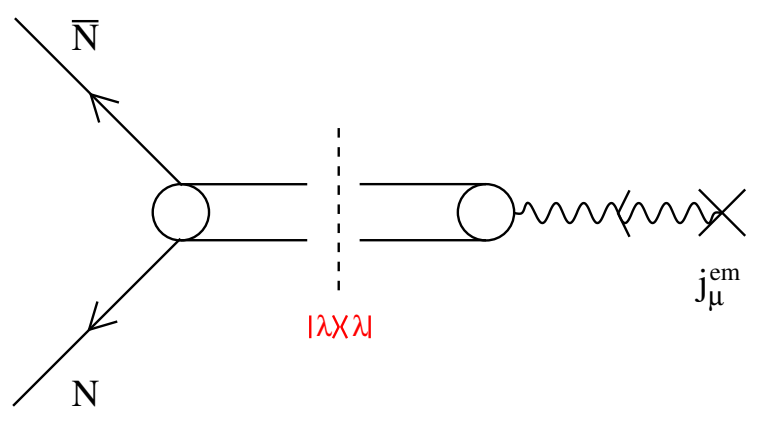

Fig. 2. The spectral decomposition of the nucleon matrix element of the electromagnetic current $j_{\mu}^{\mathrm{em}}$.

$j_{\mu}^{\mathrm{em}}$. Furthermore, they have zero net baryon number. Because of $G$-parity, states with an odd number of pions only contribute to the isoscalar part, while states with an even number contribute to the isovector part. For the isoscalar part the lowest mass states are: $3 \pi, 5 \pi, \ldots, K \bar{K}, K \bar{K} \pi, \ldots$; for the isovector part they are: $2 \pi, 4 \pi, \ldots$.

Associated with each intermediate state is a cut starting at the corresponding threshold in $t$ and running to infinity. As a consequence, the spectral function $\operatorname{Im} F(t)$ is different from zero along the cut from $t_{0}$ to $\infty$ with $t_{0}=4(9) M_{\pi}^{2}$ for the isovector (isoscalar) case.

The spectral functions are the central quantities in the dispersion-theoretical approach. Using eqs. $(7,8)$, they can in principle be constructed from experimental data. In practice, this program can only be carried out for the lightest two-particle intermediate states $(2 \pi$ and $K \bar{K})[19$, $36,37]$.

The longest-range, and therefore at low momentum transfer most important pion cloud contribution comes from the $2 \pi$ intermediate state in the isovector form factors. A new calculation of this contribution will be discussed in the following section.

\section{Two-pion continuum}

In this section, we re-evaluate the $2 \pi$ contribution in a model-independent way [38] using the latest experimental data for the pion form factor from CMD-2 [39], KLOE [40], and SND [41].

We follow ref. [42] and express the $2 \pi$ contribution to the isovector spectral functions in terms of the pion charge form factor $F_{\pi}(t)$ and the $P$-wave $\pi \pi \rightarrow \bar{N} N$ amplitudes $f_{ \pm}^{1}(t)$. The $2 \pi$ continuum is expected to be the dominant contribution to the isovector spectral function from threshold up to masses of about $1 \mathrm{GeV}$ [42]. Here, we use the expressions

$$
\begin{aligned}
\operatorname{Im} G_{E}^{v}(t) & =\frac{q_{t}^{3}}{M \sqrt{t}} F_{\pi}(t)^{*} f_{+}^{1}(t), \\
\operatorname{Im} G_{M}^{v}(t) & =\frac{q_{t}^{3}}{\sqrt{2 t}} F_{\pi}(t)^{*} f_{-}^{1}(t),
\end{aligned}
$$

where $q_{t}=\sqrt{t / 4-M_{\pi}^{2}}$. The imaginary parts of the Dirac and Pauli Form factors can be obtained using eq. (4).

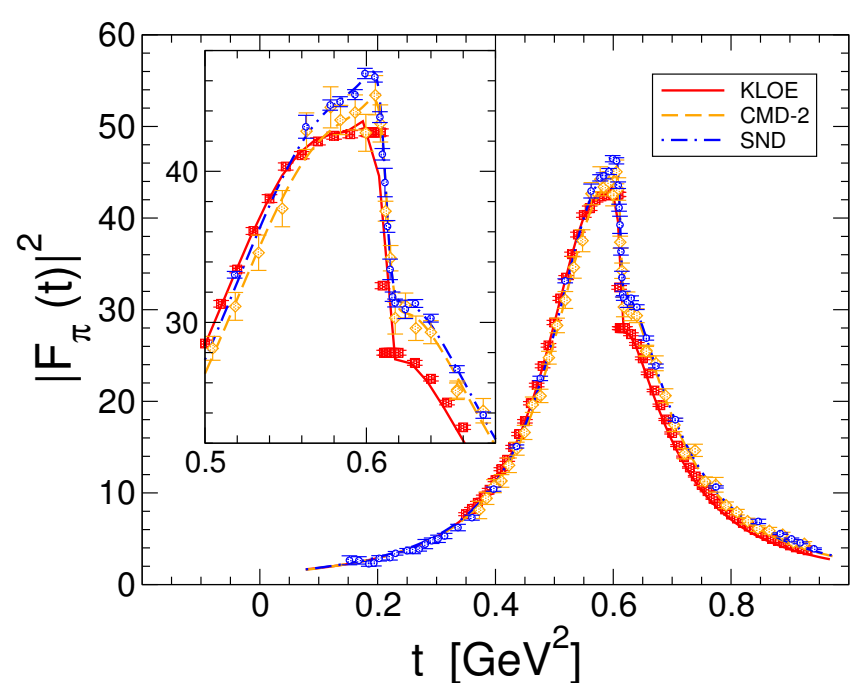

Fig. 3. The pion electromagnetic form factor $F_{\pi}(t)$ in the timelike region as a function of the momentum transfer $t$. The diamonds, squares, and circles show the high statistics data from the CMD-2 [39], KLOE [40], and SND [41] collaborations, respectively. The dashed, solid, and dash-dotted lines are our model parametrizations. The inset shows the discrepancy in the resonance region in more detail.

The $P$-wave $\pi \pi \rightarrow \bar{N} N$ amplitudes $f_{ \pm}^{1}(t)$ are tabulated in ref. [42]. (See also ref. [43] for an unpublished update that is consistent with ref. [42].) We stress that the representation of eq. (9) gives the exact isovector spectral functions for $4 M_{\pi}^{2} \leq t \leq 16 M_{\pi}^{2}$, but in practice holds up to $t \simeq 50 M_{\pi}^{2}$. Since the contributions from $4 \pi$ and higher intermediate states is small up to $t \simeq 50 M_{\pi}^{2}, F_{\pi}(t)$ and the $f_{ \pm}^{1}(t)$ share the same phase in this region and the two quantities can be replaced by their absolute values. ${ }^{2}$

The updated pion form factor is shown in fig. 3. The diamonds, squares, and circles show the high statistics data from the CMD-2 [39], KLOE [40], and SND [41] collaborations, respectively. The dashed, solid, and dashdotted lines are our model parametrizations which are of the Gounaris-Sakurai type $[20,30]$. The form factor shows a pronounced $\rho-\omega$ mixing in the vicinity of the $\rho$-peak. There are discrepancies between the three experimental data sets for the pion form factor [41]. The discrepancies in the $\rho$-resonance region are shown in more detail in the inset of fig. 3. Since we are not in the position to settle this experimental problem, we will take the three data sets at face value. We will evaluate the $2 \pi$ continuum given by eq. (9) for all three sets and estimate the errors from the discrepancy between the sets.

Using the new high statistics pion form factor data [39, 40,41] and the amplitudes $f_{ \pm}^{1}(t)$ tabulated in ref. [42], we obtain the spectral functions shown in fig. 4 [38]. We show the spectral functions weighted by $1 / t^{2}$ for $G_{E}$ (solid

\footnotetext{
${ }^{2}$ We note that representation of eq. (9) is most useful for our purpose. The manifestly real functions $J_{ \pm}(t)=f_{ \pm}^{1}(t) / F_{\pi}(t)$ also tabulated in ref. [42] contain assumptions about the pion form factor which leads to inconsistencies when used together with the updated $F_{\pi}(t)$.
} 


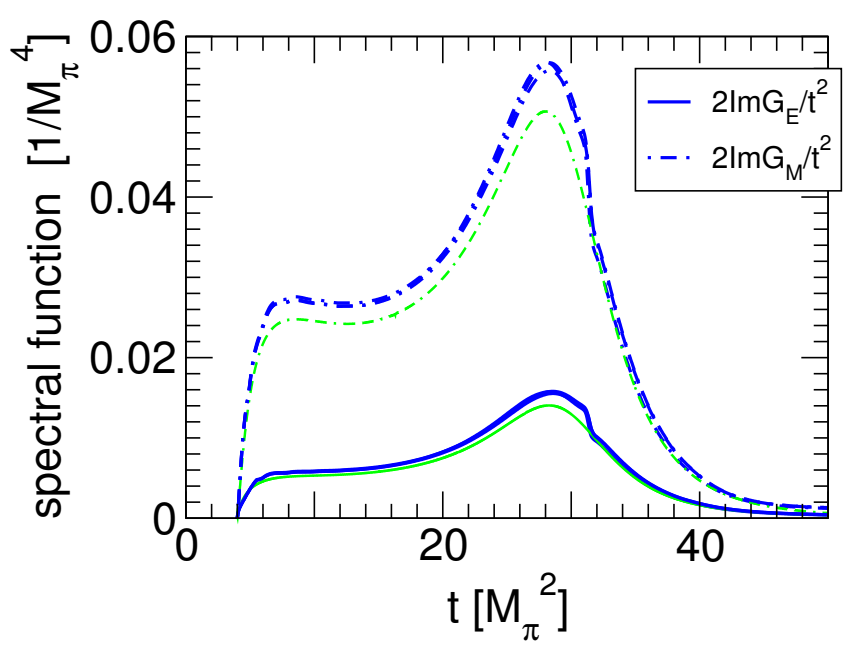

Fig. 4. The $2 \pi$ spectral function using the new high statistics data for the pion form factor $[39,40,41]$. The spectral functions weighted by $1 / t^{2}$ are shown for $G_{E}$ (solid line) and $G_{M}$ (dashdotted line) in units of $1 / M_{\pi}^{4}$. The previous results by Höhler et al. [42] (without $\rho-\omega$ mixing) are shown for comparison by the green lines.

line) and $G_{M}$ (dash-dotted line). The previous results by Höhler et al. [42] (without $\rho$ - $\omega$ mixing) are given for comparison by the gray/green lines. The general structure of the two evaluations is the same, but there is a difference in magnitude of about $10 \%$. The difference between the three data sets for the pion form factor is very small and indicated by the line thickness. The difference in the form factors is largest in the $\rho$-peak region (cf. fig. 3), but this region is suppressed by the $\pi \pi \rightarrow \bar{N} N$ amplitudes $f_{ \pm}^{1}(t)$ which show a strong fall-off as $t$ increases.

The spectral functions have two distinct features. First, as already pointed out in [15], they contain the important contribution of the $\rho$-meson with its peak at $t \simeq 30 M_{\pi}^{2}$. Second, on the left shoulder of the $\rho$, the isovector spectral functions display a very pronounced enhancement close to the two-pion threshold. This is due to the logarithmic singularity on the second Riemann sheet located at $t_{c}=4 M_{\pi}^{2}-M_{\pi}^{4} / M^{2}=3.98 M_{\pi}^{2}$, very close to the threshold. This pole comes from the projection of the nucleon Born graphs, or in modern language, from the triangle diagram.

If one were to neglect this important unitarity correction, one would severely underestimate the nucleon isovector radii [17],

$$
\left\langle r^{2}\right\rangle_{i}^{v}=\frac{6}{\pi} \int_{4 M_{\pi}^{2}}^{\infty} \frac{\mathrm{d} t}{t^{2}} \operatorname{Im} G_{i}^{v}(t),
$$

where $i=E, M$. In fact, precisely the same effect is obtained at leading one-loop accuracy in relativistic chiral perturbation theory $[44,45]$. This topic was also discussed in heavy baryon ChPT [25,27] and in a covariant calculation based on infrared regularization [26]. Thus, the most important $2 \pi$ contribution to the nucleon form factors can be determined by using either unitarity or ChPT (in the latter case, of course, the $\rho$ contribution is not included).
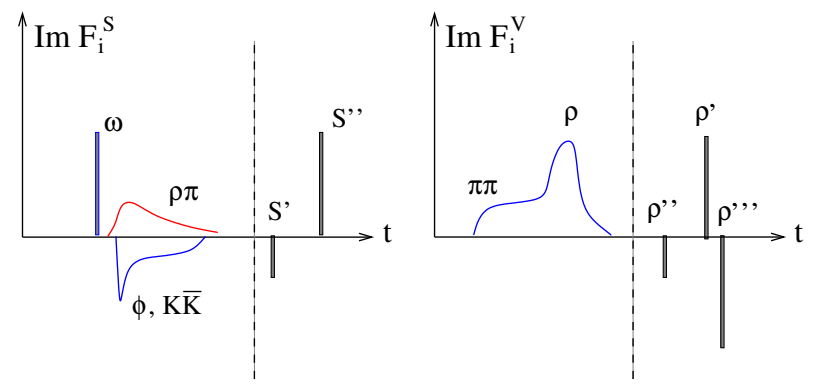

Fig. 5. Illustration of the spectral function used in the dispersion analysis. The vertical dashed line separates the well-known low-mass contributions $(2 \pi, K \bar{K}$, and $\rho \pi$ continua as well as the $\omega$ pole) from the effective poles at higher momentum transfers.

\section{Spectral functions}

As discussed above the spectral function can at present only be obtained from unitarity arguments for the lightest two-particle intermediate states $(2 \pi$ and $K \bar{K})[19,36,37]$. The $\rho \pi$ continuum contribution can be obtained from the Bonn-Jülich model [46].

The remaining contributions can be parametrized by vector meson poles. On one hand, the lower mass poles can be identified with physical vector mesons such as the $\omega$ and the $\phi$. In the the case of the $3 \pi$ continuum, e.g., it has been shown in ChPT that the nonresonant contribution is very small and the spectral function is dominated by the $\omega$ [25]. The higher mass poles on the other hand, are simply an effective way to parametrize higher mass strength in the spectral function.

For our current best fit, the spectral function includes the $2 \pi, K \bar{K}$, and $\rho \pi$ continua from unitarity and the $\omega$ pole. In addition to that there are a number of effective poles at higher momentum transfers in both the isoscalar and isovector channels. The spectral function then has the general structure

$$
\begin{aligned}
\operatorname{Im} F_{i}^{s}(t)= & \operatorname{Im} F_{i}^{K \bar{K}}(t)+\operatorname{Im} F_{i}^{\rho \pi}(t) \\
& +\sum_{V=\omega, s_{1}, \ldots} \pi a_{i}^{V} \delta\left(M_{V}^{2}-t\right), \quad i=1,2, \\
\operatorname{Im} F_{i}^{v}(t)= & \operatorname{Im} F_{i}^{2 \pi}(t) \\
& +\sum_{V=v_{1}, \ldots} \pi a_{i}^{V} \delta\left(M_{V}^{2}-t\right), \quad i=1,2,
\end{aligned}
$$

which is illustrated in in fig. 5. The vertical dashed line separates the well-known low-mass contributions to the spectral function from the effective poles at higher momentum transfers.

In our fits, we also include the widths of the vector mesons. The width and mass of the $\omega$ are taken from the particle data tables while the masses and widths of the effective poles are fitted to the form factor data. We have performed various fits with different numbers of effective poles and including/excluding some of the continuum contributions. In sect. 7 , we will discuss preliminary results of this ongoing effort. 


\section{Constraints}

The number of parameters in the fit function is reduced by enforcing various constraints. The first set of constraints concerns the low- $t$ behavior of the form factors. First, we enforce the correct normalization of the form factors, which is given in eq. (2). Second, we constrain the neutron radius from a low-energy neutron-atom scattering experiment $[47,48]$.

Perturbative QCD (pQCD) constrains the behavior of the nucleon em form factors for large momentum transfer. Brodsky and Lepage [49] find for $t \rightarrow-\infty$,

$$
F_{i}(t) \rightarrow(-t)^{-(i+1)}\left[\ln \left(\frac{-t}{Q_{0}^{2}}\right)\right]^{-\gamma}, \quad i=1,2,
$$

where $Q_{0} \simeq \Lambda_{\mathrm{QCD}}$. The anomalous dimension $\gamma$ depends weakly on the number of flavors, $\gamma=2.148,2.160,2.173$ for $N_{f}=3,4,5$, in order.

The power behavior of the form factors at large $t$ can be easily understood from perturbative gluon exchange. In order to distribute the momentum transfer from the virtual photon to all three quarks in the nucleon, at least two massless gluons have to be exchanged. Since each of the gluons has a propagator $\sim 1 / t$, the form factor has to fall off as $1 / t^{2}$. In the case of $F_{2}$, there is additional suppression by $1 / t$ since a quark spin has to be flipped. The power behavior of the form factors leads to superconvergence relations of the form

$$
\int_{t_{0}}^{\infty} \operatorname{Im} F_{i}(t) t^{n} \mathrm{~d} t=0
$$

with $n=0$ for $F_{1}$ and $n=0,1$ for $F_{2}$. The asymptotic behavior of eq. (13) is obtained by choosing the residues of the vector meson pole terms such that the leading terms in the $1 / t$-expansion cancel.

The logarithmic term in eq. (13) was included in some of our earlier analyses [20,21,23] but has little impact on the fit. The particular way this constraint was implemented, however, lead to an unphysical logarithmic singularity of the form factors in the time-like region. In order to be able to include the data for the form factors at large time-like momentum transfers, the logarithmic constraint is not enforced in the current analysis.

The number of effective poles in eqs. $(11,12)$ is determined by the stability criterion discussed in detail in [50]. In short, we take the minimum number of poles necessary to fit the data. For the preliminary results discussed in the next section, we took 4 effective isoscalar poles and 3 effective isovector poles whose residua, masses, and widths are fitted to the data. The number of free parameters is strongly reduced by the various constraints (unitarity, normalizations, superconvergence relations), so that we end up with 19 free parameters in the preliminary fit presented in the next section. Our general strategy is to reduce the number of parameters even further without sacrificing the quality of the fit.

\section{Fit results}

We now discuss some preliminary fit results that are representative for the current status of the analysis. We present results for a fit with 4 effective isoscalar poles and 3 effective isovector poles whose residua, masses, and widths are fitted to the data.

In fig. 6, we show the results for all four form factors compared to the world data for the form factors. Our data basis is taken from ref. [34] and in addition also includes the new data that have appeared since 2003 (see ref. [5]). The results for $G_{M}^{n}, G_{E}^{p}, G_{M}^{p}$ are normalized to the phenomenological dipole fit:

$$
G_{D}\left(Q^{2}\right)=\left(1+\frac{Q^{2}}{m_{D}^{2}}\right)^{-2},
$$

where $m_{D}^{2}=0.71 \mathrm{GeV}^{2}$. The dash-dotted line gives the result of ref. [23], while the the solid line indicates our present best fit. The new fit leads to an improved description of the form factor data compared with ref. [23]. In particular, the rapid fall-off of the JLab polarization data for $G_{E}^{p}[51,52]$ is now described. The $\chi^{2}$ per degree of freedom is 0.84 . Note that we do not obtain a pronounced bump structure in $G_{E}^{n}$ as observed in ref. [34]. We will come back to this question in sect. 9 and discuss the modifications in the spectral function required to produce this structure.

The stability constraint requires to use the minimum number of poles required to describe the data [50]. In the future, we plan to further reduce the number of effective poles in order to improve the stability.

Table 1. Nucleon radii in fm extracted from the fit in fig. 6 .

\begin{tabular}{|c|c|c|c|}
\hline & This work & Ref. [23] & Recent determ. \\
\hline \hline$r_{E}^{p}[\mathrm{fm}]$ & $0.84 \ldots 0.857$ & 0.848 & $0.886(15)[53,54,55]$ \\
$r_{M}^{p}[\mathrm{fm}]$ & $0.85 \ldots 0.875$ & 0.857 & $0.855(35)[54,56]$ \\
$r_{E}^{n}[\mathrm{fm}]$ & $-0.12 \ldots-0.10$ & -0.12 & $-0.115(4)[48]$ \\
$r_{M}^{n}[\mathrm{fm}]$ & $0.86 \ldots 0.88$ & 0.879 & $0.873(11)[57]$ \\
\hline
\end{tabular}

In Table 1, we give the nucleon radii extracted from our fit. The neutron radius is included as a soft constraint in our fit and therefore not a prediction. ${ }^{3}$ The other nucleon radii are generally in good agreement with other recent determinations using only low-momentum-transfer data given in the table. Our result for the proton radius, however, is somewhat small. This was already the case in the dispersion analyses of refs. [20,23]. We speculate that the reason for this discrepancy lies in inconsistencies in the data sets. In this type of global analysis all four form factors are analyzed simultaneously and both data at small and large momentum transfers enter. This can be an advantage or a disadvantage depending on the question at hand. Another possible reason for the discrepancy is $2 \gamma$ physics which was neglected in the data analysis of most older experiments [58].

\footnotetext{
3 A soft constraint is not implemented exactly but deviations from the constraint are penalized in the $\chi^{2}$ of the fit.
} 

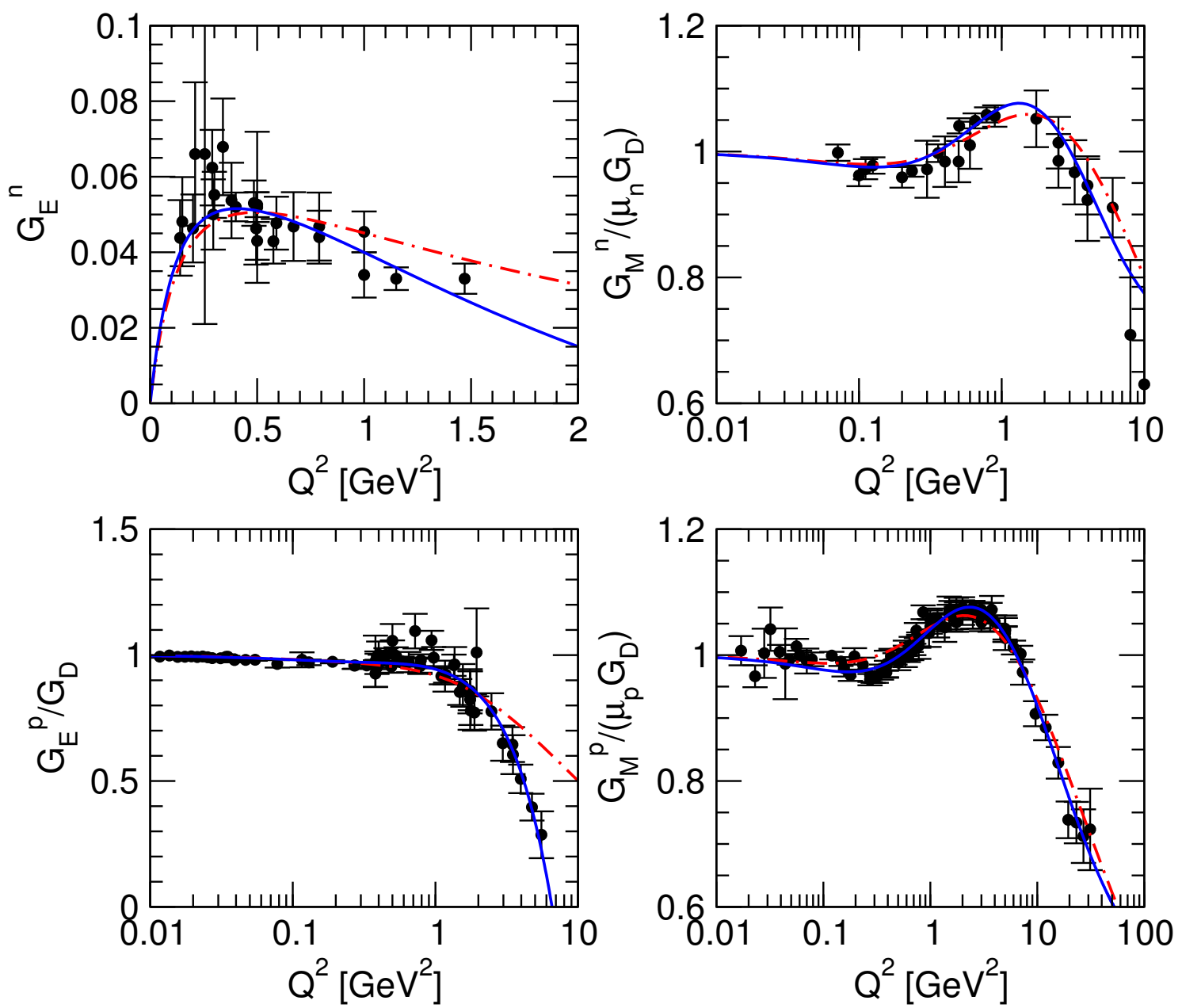

Fig. 6. The nucleon electromagnetic form factors for space-like momentum transfer. The results for $G_{M}^{n}, G_{E}^{p}, G_{M}^{p}$ are normalized to the dipole fit. The dash-dotted line gives the result of ref. [23], while the the solid line indicates our preliminary best fit.

\section{Time-like data}

We have also performed first fits that include data in the time-like region. The extraction of these data is more challenging than in the space-like region. At the nucleonantinucleon threshold, the electric and magnetic form factors are equal by definition: $G_{M}\left(4 M^{2}\right)=G_{E}\left(4 M^{2}\right)$, while one expects the magnetic form factor to dominate at large momentum transfer. Moreover, the form factors are complex in the time-like region, since several physical thresholds are open. Separating $\left|G_{M}\right|$ and $\left|G_{E}\right|$ unambiguously from the data requires a measurement of the angular distribution, which is difficult. In most experiments, it has been assumed that either $\left|G_{M}\right|=\left|G_{E}\right|$ (which should be a good approximation close to the two-nucleon threshold) or $\left|G_{E}\right|=0$ (which should be a good approximation for large momentum transfers). Most recent data have been presented using the latter hypothesis.

The time-like data were already included in the dispersion analyses of refs. [21,22]. The proton magnetic form factor up to $t \approx 6 \mathrm{GeV}^{2}$ was well described by these analyses. Data at higher momentum transfers were not included. The data for the neutron magnetic form factor are from the pioneering FENICE experiment [11]. They have been analyzed under both the assumption $\left|G_{E}\right|=\left|G_{M}\right|$ and $\left|G_{E}\right|=0$. The latter hypothesis is favored by the measured angular distributions [11]. Neither data set could be described by the analysis [22].

In fig. 7, we show the current status of the analysis of the time-like data for the magnetic form factors. For the proton magnetic form factor, data up to momentum transfers $t \approx 15 \mathrm{GeV}^{2}$ have been included $[6,7,8,9,10]$. Our preliminary fit gives a good description in the threshold region but starts to deviate significantly around $t \approx 5 \mathrm{GeV}^{2}$. The data for $t \geq 10 \mathrm{GeV}^{2}$ are well described. This seems to be due to a slight inconsistency in the data around $5 \mathrm{GeV}^{2}$ and for $t \geq 10 \mathrm{GeV}^{2}$. This question deserves further attention.

The status for the neutron form factor is the same as in the previous analysis [22]: Neither of the two data sets from ref. [11] can be described. Even though we are not yet in the region where perturbative QCD is applicable, it comes as a surprise that the neutron form factor is larger in magnitude than the proton one. Perturbative QCD predicts asymptotically equal magnitudes. In any case, there is interesting physics in the time-like nucleon form factors 

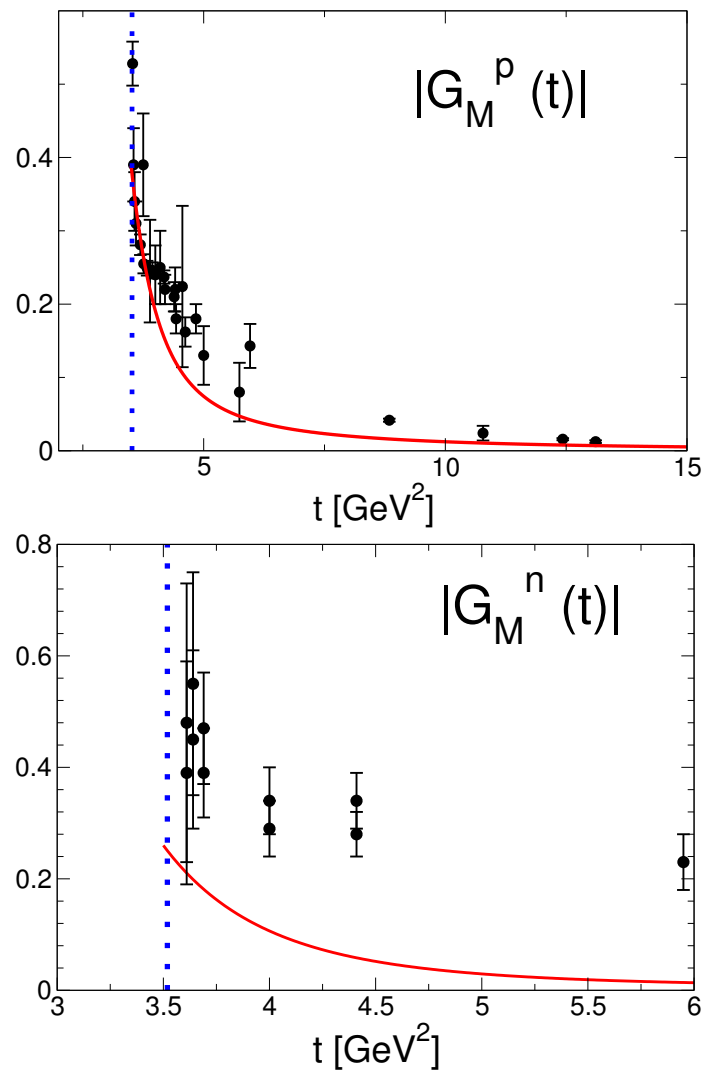

Fig. 7. Current status of our analysis of the magnetic form factors in the time-like region compared to the world data [6, $7,8,9,10,11]$. The solid line gives our preliminary best fit, while the vertical dotted line indicates the two-nucleon threshold.

and new precision experiments such as the PANDA and PAX experiments at GSI would be very welcome.

\section{Pion cloud of the nucleon}

Friedrich and Walcher (FW) recently analysed the em nucleon form factors and performed various phenomenological fits [34]. Their fits showed a pronounced bump structure in $G_{E}^{n}$ which they interpreted using an ansatz for the pion cloud based on the idea that the proton can be thought of as a virtual neutron-positively charged pion pair. They found a very long-range contribution to the charge distribution in the Breit frame extending out to about $2 \mathrm{fm}$ which they attributed to the pion cloud. While naively the pion Compton wave length is of this size, these findings are indeed surprising if compared with the "pion cloud" contribution due to the $2 \pi$ continuum contribution to the isovector spectral functions discussed in sect. 4 .

As was shown by Hammer, Drechsel, and Meißner [35], these latter contributions to the long-range part of the nucleon structure are much more confined in coordinate space and agree well with earlier (but less systematic) calculations based on chiral soliton models, see, e.g., [59]. In the dispersion-theoretical framework, the longest-range part of the pion cloud contribution to the nucleon form

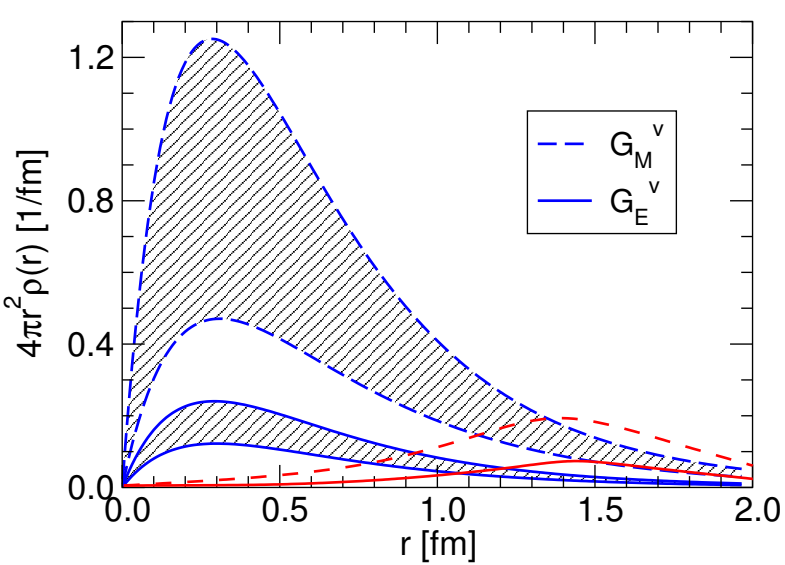

Fig. 8. Pion cloud contribution to the nucleon charge density. The lines show the result of Friedrich and Walcher [34], while the bands give the result of ref. [35]. Only the long-range contributions for $r \gtrsim 1 \mathrm{fm}$ are meaningful for the comparison of the two results.

factors is given by the $2 \pi$ continuum - the lowest-mass intermediate state including only pions. Note that a onepion intermediate state is forbidden by parity.

The nonresonant part of the $2 \pi$ continuum can be calculated in ChPT [27] while the full continuum can be obtained from experimental data and unitarity as discussed in sect. 4. The "pion cloud" corresponds to the nonresonant part of the $2 \pi$ continuum excluding the $\rho$. Consequently, the $\rho$ contribution has to be subtracted from the full $2 \pi$ continuum. ${ }^{4}$ The error in this subtraction was estimated using three different methods for the separation of the contributions [35].

The charge distribution can then be obtained from the nonresonant part of the $2 \pi$ continuum by Fourier transformation. This leads to the relation:

$$
\rho_{i}^{v}(r)=\frac{1}{4 \pi^{2}} \int_{4 M_{\pi}^{2}}^{40 M_{\pi}^{2}} \mathrm{~d} t \operatorname{Im} G_{i}^{v}(t) \frac{e^{-r \sqrt{t}}}{r},
$$

where $i=E, M$. The $2 \pi$ contribution from $t \geq 40 M_{\pi}^{2}$ is small and can be neglected [35].

The result for the pion cloud contribution to the nucleon charge density is shown in fig. 8. The lines show the result of FW [34], while the bands give the result of ref. [35]. Only the long-range contributions for $r \gtrsim 1 \mathrm{fm}$ should be compared since the separation of the shortrange part into resonant and nonresonant contributions is arbitrary. In comparison with ref. [34], the $2 \pi$ continuum contribution to the charge density is generally much smaller at distances beyond $1 \mathrm{fm}, e . g$., by a factor of 3 for $\rho_{E}^{v}(r)$ at $r=1.5 \mathrm{fm}$. We emphasize that this result is obtained from independent physical information that determines the $2 \pi$ continuum (pion form factor and $\pi \pi \rightarrow N \bar{N}$ amplitudes, cf. sect. 4) and not from form factor fits.

As a consequence, it remains to be shown how the proposed long-range pion cloud can be reconciled with what

4 Note that this separation is not unique. It is only meaningful for the long-range part. The separation of the short-range part is model- and even representation-dependent. 


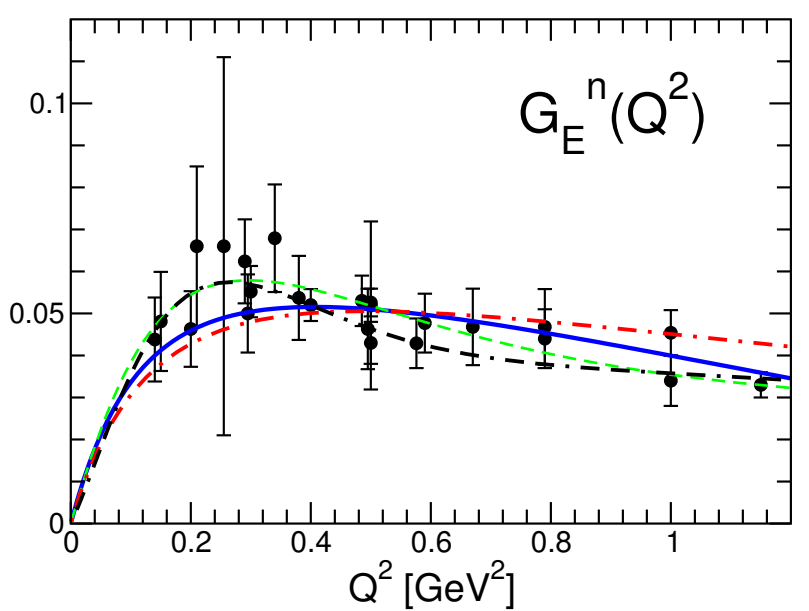

Fig. 9. The neutron electric form factor at low momentum transfer: present fit with additional low-mass strength (dashed line) compared to the fits of Friedrich and Walcher [34] (doubledash-dotted line). For comparison, the fits of sect. 7 (solid line) and ref. [23] (dash-dotted line) are also shown.

is known from dispersion relations and ChPT. In order to clarify this issue, we have performed various fits in order to understand what structures in the spectral function are required to reproduce the bump in $G_{E}^{n}$. We find that the structure can only be reproduced if additional low-mass strength in the spectral function below $t \lesssim 1 \mathrm{GeV}^{2}$ is allowed beyond the $2 \pi, K \bar{K}$, and $\rho \pi$ continua and the $\omega$ pole. In the fits of sects. 7 and 8 such strength was explicitly excluded.

In fig. 9, we show the neutron electric form factor at low momentum transfer. The fit of FW [34] is given by the double-dash-dotted line, while the present fit with additional low-mass strength is given by the dashed line. For comparison, we show also the fit of ref. [23] (dashdotted line) and the fit from sect. 7 (solid line). The fit with additional low-mass strength shows a clear bump structure around $Q^{2} \sim 0.3 \mathrm{GeV}^{2}$. This structure requires three additional low mass poles: two isoscalar poles at $M_{s}^{2}=0.13 \mathrm{GeV}^{2}, 0.54 \mathrm{GeV}^{2}$ and one isovector pole at $M_{v}^{2}=0.30 \mathrm{GeV}^{2}$. In principle, vector meson dominance works well for $t \leq 1 \mathrm{GeV}^{2}$ and one should be able to interpret these poles as physical vector mesons. However, no such vector mesons are known in this region. This raises the question of whether the effective low-mass poles can be interpreted as something else?

One possible solution would be to interpret the poles as effective poles mimicking some continuum contribution. It is interesting to note that the three low-mass poles happen to come out at the thresholds of the $3 \pi, 4 \pi$ and $5 \pi$ continua and are located in the correct isospin channel. Maybe these higher-order pion continua are more important than previously thought and have a threshold enhancement similar to the $2 \pi$ continuum that is accounted for by the effective poles?

Even though this scenario has a certain appeal, it appears unlikely given the current state of knowledge. In ref. [25], the threshold behavior of the $3 \pi$ continuum was explicitly calculated in heavy baryon ChPT and no enhancement was found. Moreover, the inelasticity from four pions in $\pi \pi$ scattering and four-pion production in $e^{+} e^{-}$ annihilation at low momentum transfer are known to be small $[42,60,61]$.

\section{Summary \& outlook}

Dispersion theory simultaneously describes all four nucleon form factors over the whole range of momentum transfers in both the space-like and time-like regions. It allows for the inclusion of constraints from other physical processes, unitarity, and ChPT and therefore is an ideal tool to analyze the form factor data.

We have presented preliminary results for our new dispersion analysis that is currently carried out in Bonn. The spectral function has been improved and contains the updated $2 \pi$ continuum [38], as well the $K \bar{K}[36,37]$ and $\rho \pi$ continua [46]. Our preliminary best fit gives a consistent description of the world data in the space-like region. The understanding of the time-like form factors is more difficult and a future challenge for theorists and experimentalists alike.

As part of this ongoing theoretical program, many things remain to be done: The stability constraint requires to use the minimum number of poles. Our strategy for the future is to successively reduce the number of poles without sacrifying the quality of the fit. Furthermore, the description of the time-like data needs to be improved. In previous experiments, the separation of $G_{E}$ and $G_{M}$ could only be carried out under overly simplifying assumptions. New data, such as planned for the PANDA and PAX experiments at GSI, are therefore called for.

Other improvements concern the quantification of theoretical and systematic uncertainties in the analysis, the inclusion of perturbative QCD corrections beyond superconvergence (leading logarithms etc.), and the inclusion of two-photon physics. The latter point might require to analyze the cross section data directly. Last but not least, the consequences of the new data for the strange vector form factors of the nucleon need to be worked out.

This work was done in collaboration with M.B. Belushkin, D. Drechsel, and Ulf-G. Meißner. M.J. Ramsey-Musolf has contributed in earlier stages of the project. The work was supported in part by the EU I3HP under contract number RII3CT-2004-506078 and the DFG through funds provided to the SFB/TR 16 "Subnuclear Structure of Matter" and SFB 443 "Many Body Structure of Strongly Interacting Systems". I would like to thank Hartmuth Arenhövel, Hartmut Backe, Dieter Drechsel, Jörg Friedrich, Karl-Heinz Kaiser, and Thomas Walcher for a very stimulating and enjoyable time in Mainz. I have had many personal interactions with them through scientific discussions and/or through lectures and seminars I attended as a student. In particular, I want to thank my $\mathrm{PhD}$ advisor Dieter Drechsel from whom I have learned much about physics and research. 


\section{References}

1. H. Gao, Int. J. Mod. Phys. E 12, 1 (2003); 12, 567 (2003)(E) (arXiv:nucl-ex/0301002).

2. C.E. Hyde-Wright, K. de Jager, Annu. Rev. Nucl. Part. Sci. 54, 217 (2004) (arXiv:nucl-ex/0507001).

3. Th. Udem et al., Phys. Rev. Lett. 79, 2646 (1997).

4. D.H. Beck, B.R. Holstein, Int. J. Mod. Phys. E 10, 1 (2001) (arXiv:hep-ph/0102053).

5. M. Ostrick, these proceedings and references therein.

6. E835 Collaboration (M. Ambrogiani et al.), Phys. Rev. D 60, 032002 (1999).

7. BES Collaboration (M. Ablikim et al.), Phys. Lett. B 630 , 14 (2005) (arXiv:hep-ex/0506059).

8. CLEO Collaboration (T.K. Pedlar et al.), Phys. Rev. Lett. 95, 261803 (2005) (arXiv:hep-ex/0510005).

9. BABAR Collaboration (B. Aubert et al.), Phys. Rev. D 73, 012005 (2006) (arXiv:hep-ex/0512023).

10. R. Baldini, E. Pasqualucci, in Chiral Dynamics: Theory and Experiment, edited by A.M. Bernstein, B.R. Holstein, Lect. Notes Phys., Vol. 452 (Springer, Heidelberg, 1995).

11. A. Antonelli et al., Nucl. Phys. B 517, 3 (1998).

12. H. Fröhlich, W. Heitler, N. Kemmer, Proc. R. Soc. A 166, 155 (1938).

13. G.F. Chew, R. Karplus, S. Gasiorowicz, F. Zachariasen, Phys. Rev. 110, 265 (1958).

14. P. Federbush, M.L. Goldberger, S.B. Treiman, Phys. Rev. 112, 642 (1958).

15. W.R. Frazer, J.R. Fulco, Phys. Rev. Lett. 2, 365 (1959).

16. W.R. Frazer, J.R. Fulco, Phys. Rev. 117, 1609 (1960).

17. G. Höhler, E. Pietarinen, Phys. Lett. B 53, 471 (1975).

18. G. Höhler et al., Nucl. Phys. B 114, 505 (1976).

19. G. Höhler, E. Pietarinen, Nucl. Phys. B 95, 210 (1975).

20. P. Mergell, U.-G. Meißner, D. Drechsel, Nucl. Phys. A 596, 367 (1996) (arXiv:hep-ph/9506375).

21. H.-W. Hammer, U.-G. Meißner, D. Drechsel, Phys. Lett. B 385, 343 (1996) (arXiv:hep-ph/9604294).

22. H.-W. Hammer, in Proceedings of the $e^{+} e^{-}$Physics at Intermediate Energies Conference, edited by Diego Bettoni, eConf C010430, W08 (2001) (arXiv:hep-ph/0105337).

23. H.-W. Hammer, U.-G. Meißner, Eur. Phys. J. A 20, 469 (2004) (arXiv:hep-ph/0312081)

24. V. Bernard, N. Kaiser, U.-G. Meißner, Int. J. Mod. Phys. E 4, 193 (1995) (arXiv:hep-ph/9501384).

25. V. Bernard, N. Kaiser, U.-G. Meißner, Nucl. Phys. A 611, 429 (1996) (arXiv:hep-ph/9607428).

26. B. Kubis, U.-G. Meißner, Nucl. Phys. A 679, 698 (2001) (arXiv:hep-ph/0007056).

27. N. Kaiser, Phys. Rev. C 68, 025202 (2003) (arXiv:nuclth/0302072).

28. M.R. Schindler, J. Gegelia, S. Scherer, Eur. Phys. J. A 26 , 1 (2005) (arXiv:nucl-th/0509005).

29. J.J. Sakurai, Ann. Phys. (NY) 11, 1 (1960).

30. G.J. Gounaris, J.J. Sakurai, Phys. Rev. Lett. 21, 244 (1968).

31. M. Gari, W. Krümpelmann, Z. Phys. A 322, 689 (1985).

32. E.L. Lomon, Phys. Rev. C 64, 035204 (2001) (arXiv:nuclth/0104039).
33. S. Dubnicka, A.Z. Dubnickova, P. Weisenpacher, J. Phys. G 29, 405 (2003) (arXiv:hep-ph/0208051).

34. J. Friedrich, T. Walcher, Eur. Phys. J. A 17, 607 (2003) (arXiv:hep-ph/0303054).

35. H.-W. Hammer, D. Drechsel, U.-G. Meißner, Phys. Lett. B 586, 291 (2004) (arXiv:hep-ph/0310240).

36. H.-W. Hammer, M.J. Ramsey-Musolf, Phys. Rev. C 60, 045205 (1999); 62, 049903 (2000)(E) (arXiv:hep$\mathrm{ph} / 9812261)$.

37. H.-W. Hammer, M.J. Ramsey-Musolf, Phys. Rev. C 60, 045204 (1999); 62, 049902 (2000)(E) (arXiv:hep$\mathrm{ph} / 9903367)$.

38. M.A. Belushkin, H.-W. Hammer, U.-G. Meißner, Phys. Lett. B 633, 507 (2006) (arXiv:hep-ph/0510382).

39. CMD-2 Collaboration (R.R. Akhmetshin et al.), arXiv:hep-ex/9904027; Phys. Lett. B 527, 161 (2002) (arXiv:hep-ex/0112031); 578, 285 (2004) (arXiv:hepex/0308008).

40. KLOE Collaboration (A. Aloisio et al.), Phys. Lett. B 606 , 12 (2005) (arXiv:hep-ex/0407048).

41. M.N. Achasov et al., J. Exp. Theor. Phys. 101, 1053 (2005) (arXiv:hep-ex/0506076).

42. G. Höhler, Pion-Nucleon Scattering, Landolt-Börnstein Vol. I/9b, edited by H. Schopper (Springer, Berlin, 1983).

43. E. Pietarinen, A calculation of $\pi \pi \rightarrow N \bar{N}$ amplitudes in the pseudophysical region, University of Helsinki Preprint Series in Theoretical Physics, HU-TFT-17-77, unpublished.

44. J. Gasser, M.E. Sainio, A. Svarc, Nucl. Phys. B 307, 779 (1988).

45. U.-G. Meißner, Int. J. Mod. Phys. E 1, 561 (1992).

46. U.-G. Meißner, V. Mull, J. Speth, J.W. van Orden, Phys. Lett. B 408, 381 (1997) (arXiv:hep-ph/9701296).

47. S. Kopecky et al., Phys. Rev. Lett. 74, 2427 (1995).

48. S. Kopecky, M. Krenn, P. Riehs, S. Steiner, J.A. Harvey, N.W. Hill, M. Pernicka, Phys. Rev. C 56, 2229 (1997).

49. S.J. Brodsky, G.P. Lepage, Phys. Rev. D 22, 2157 (1980).

50. I. Sabba-Stefanescu, J. Math. Phys. 21, 175 (1980).

51. Jefferson Lab Hall A Collaboration (M.K. Jones et al.), Phys. Rev. Lett. 84, 1398 (2000) (arXiv:nucl-ex/9910005).

52. Jefferson Lab Hall A Collaboration (O. Gayou et al.), Phys. Rev. Lett. 88, 092301 (2002) (arXiv:nuclex/0111010).

53. R. Rosenfelder, Phys. Lett. B 479, 381 (2000) (arXiv:nuclth/9912031)

54. I. Sick, Phys. Lett. B 576, 62 (2003) (arXiv:nuclex/0310008).

55. K. Melnikov, T. van Ritbergen, Phys. Rev. Lett. 84, 1673 (2000) (arXiv:hep-ph/9911277).

56. I. Sick, private communication.

57. G. Kubon et al., Phys. Lett. B 524, 26 (2002) (arXiv:nuclex/0107016).

58. P.A.M. Guichon, M. Vanderhaeghen, Phys. Rev. Lett. 91, 142303 (2003) (arXiv:hep-ph/0306007).

59. U.-G. Meißner, Phys. Rep. 161, 213 (1988).

60. J. Gasser, U.-G. Meißner, Nucl. Phys. B 357, 90 (1991).

61. G. Ecker, R. Unterdorfer, Eur. Phys. J. C 24, 535 (2002) (arXiv:hep-ph/0203075). 